\title{
Biochemical measurements: summary
}

\author{
R. F. Addison \\ Bedford Institute of Oceanography, PO Box 1006, Dartmouth, Nova Scotia, Candda B2Y 4A2
}

It was recognised at the planning stage that the Bremerhaven Workshop was held at the least desirable time for biochemical studies on the dab. Previous experimental and field studies had shown that the stresses caused by reproduction in fish were likely to confound any responses attributable to contamination. (Most programmes which include biochemical effects measurements require that samples be taken outside the reproductive season, and that they be restricted to immature males.) The fact that many of the biochemical measurements on pre-spawning female dab detected 'signals' due to contamination in the presence of the 'noise' due to spawning indicates the strength of these techniques.

As in previous workshops, the approaches used at Bremerhaven ranged from established methods [e.g. mixed function oxidase (MFO) induction] to exploratory techniques, such as measurement of antioxidant enzyme activity. These included:

- MFO (or mono-oxygenase) induction in dab Limanda limanda liver and other tissues;

- Metallothionein (MT) induction in dab liver and gill;

- Acetyl- and butyryl-cholinesterase (AChE and $\mathrm{BChE}$ ) inhibition in dab muscle;

- ATPase inhibition in dab gill;

- Antioxidant enzyme activity in dab liver.

MFO induction. MFOs are induced by a limited suite of organic compounds which includes polynuclear aromatic hydrocarbons (PAH) and co-planar congeners of polychlorinated biphenyls (PCB), chlorinated dibenzodioxins (CDD) and dibenzofurans (CDF). The biochemistry of MFO induction is well understood and the links between the presence of a chemical in the environment and induction are well defined. MFO induction therefore reliably indicates organic contamination, and this was demonstrated once again at Bremerhaven.

Standard measurements of MFO induction include hepatic EROD (ethoxyresorufin O-de-ethylase) activ- ity and immunochemical measurement of hepatic P-450 1A1. Both were measured at the workshop, and showed that fish caught inshore (Stns 1, 3, \& 5) had induced MFO systems; those from offshore (Stns 6 to 8) had lower MFO activities, and there appeared to be a slight increase at Stn 9 in the Dogger Bank. MFO induction was well correlated with tissue organochlorine residue concentrations, and with some sediment hydrocarbon concentrations. Fish from the drilling site transect showed no evidence of induction; indices of MFO activity there were similar to those from the offshore stations at the German Bight. It should be emphasised that these analyses were carried out by 3 different groups of investigators, using different techniques - including an experimental approach based on a microplate reader - on different sets of fish sampled at different times. All these measurements led to the same general conclusions.

More exploratory approaches involved the measurement of EROD and P-450 1A1 from other tissues. Kidney samples from inshore fish were induced, as were livers from the same fish. Gill P-450 1A1 levels, however, were not consistent with those in liver or kidney. Other experimental approaches involved the measurement of hepatic P-450 1A1 mRNA using a new synthetic oligonucleotide probe, and of the activity of hepatic cyanoethoxycoumarin O-de-ethylase (CNECOD). P-450 1A1 mRNA concentrations were less well correlated with tissue residue concentrations than was EROD or P-450 1A1 itself; CN-ECOD activity, however, was well correlated with tissue and sediment contaminant concentrations, and appeared to be a sensitive indicator of contamination.

Overall, MFO measurements were internally consistent: $P-4501 \mathrm{~A} 1 \mathrm{mRNA}, P-4501 \mathrm{~A} 1$ itself and its catalytic activity (shown by EROD and CN-ECOD) were all well correlated in liver. Furthermore, these indices of MFO induction were well correlated with chemical residue concentrations in fish tissues or in the sediments from which the fish were caught. MFO in- 
duction appeared to be a reliable indicator of spatial variation in contamination. As noted above, this was somewhat surprising as factors such as reproductive maturity are known to affect MFO systems. In these analyses, however, the effects of reproduction and migration evidently did not obscure the MFO results.

Metallothionein induction. MT in liver is induced by metals such as $\mathrm{Cd}, \mathrm{Cu}$ and $\mathrm{Zn}$. The rationale for using MT induction to indicate the presence of these metals is similar to that for using MFO induction to indicate some organic contaminants. At the Workshop, 2 groups of investigators measured MT induction in dab liver and gill.

MT concentrations in dab liver followed several complex trends; in females, MT was best correlated with tissue $\mathrm{Zn}$ but in males it was best correlated with a combination of $\mathrm{Cd}$ and $\mathrm{Cu}$. Tissue metal concentrations in both male and female dab were more consistent with metal concentrations in invertebrates than with metal concentrations in sediments. Metal (and usually MT) concentrations were sometimes higher in livers from fish offshore than in those from close inshore. In contrast, gill $\mathrm{Zn}$ and $\mathrm{Cd}$ concentrations (in male fish) were higher inshore than offshore; $\mathrm{Cu}$ showed no clear trend in gills. Gill MT showed a generally declining trend along Stns $1,3,5,7, \& 9$.

Overall, these data suggest that the relationship between metal exposure and MT induction is complex. Metal accumulation varies between tissues, and the response of MT induction also varies between tissues and with metal exposure. MT induction probably responds to both exogenous factors (such as environmental metal exposure) and endogenous factors, which may be confounded by the process of reproduction.

Cholinesterase inhibition. The cholinesterases hydrolyse the neurotransmitter acetylcholine. The enzymes can be inhibited by covalent binding to various chemicals, including organophosphorus and carbamate pesticides. Cholinesterase inhibition in fish has often been used to assess the impact of these pesticides in freshwater systems, but it seems to have been assumed that such chemicals would not persist in marine systems, and until very recently there were no studies of inhibition in contaminant-exposed marine fish.

Acetyl-and butyryl-cholinesterase activities (AChE and $\mathrm{BChE}$ ) were measured in dab muscle from the German Bight and around the drilling site. In the German Bight, the trend in enzyme activities was similar to that seen for the impact of other organic pollutants: activities were lowest (indicating most inhibition) inshore (Stns $1 \& 3$ ), climbing at the offshore stations (Stns 5 to 8 ) and declining again slightly at Stn 9. Samples from the drilling site transect had
AChE activities similar to those at the offshore German Bight stations, suggesting relatively little contamination.

No analyses were made of the chemicals expected to inhibit cholinesterase. However, the spatial variation in cholinesterase inhibition in the German Bight samples was very similar to that in the distribution of other organic contaminants. This raises the question of whether known cholinesterase inhibitors (organophosphorus and carbamate pesticides) are more persistent in marine systems than was previously believed, or whether cholinesterase inhibitors other than these chemicals also occur as contaminants. In any case, this biochemical measurement has raised questions which must be addressed by chemical analysis.

ATPase inhibition. $\mathrm{Na}^{+}, \mathrm{K}^{+}$-ATPases are involved in osmoregulation and ion balance. Numerous studies in vitro and a few in vivo have shown them to be inhibited by a wide range of organic chemicals, including several marine contaminants. Several indices of the activity of these enzymes were measured in dab gill sampled along the German Bight transect.

Total $\mathrm{Na}^{+}, \mathrm{K}^{+}$-ATPase activity did not change along the transect, but amounts of the enzyme appeared higher at the innermost (Stn 1) and outermost (Stn 9) stations. Turnover rates of the enzyme showed a declining trend from inshore to offshore stations, with an increase again at $\operatorname{Stn} 9$. Turnover rates were usually inversely related to $P-4501 \mathrm{~A} 1$ concentrations in the gill, but were less strongly related to liver or sediment concentrations of organic contaminants.

Antioxidant enzymes. Oxyradical production is a potentially damaging response of biochemical systems exposed to some contaminants. The concentration of oxyradicals is controlled by a group of antioxidant enzymes, whose activity may therefore indicate contaminant exposure.

The indices of antioxidant enzyme activity studied showed a variable response to the contaminant gradient. Some (such as catalase and glutathione peroxidase) showed increased activity at the more contaminated sites; others such as superoxide dismutase followed trends not easily related to contaminant distribution. Although the measurement of antioxidant enzyme activity as an indicator of contaminant exposure is attractive conceptually, the response of the system to factors other than contamination must be defined.

General conclusions. (1) Hepatic MFO induction methods, including measurements of EROD and $P-4501 \mathrm{~A} 1$, proved once again to be reliable indicators of organic contaminant exposure. Hepatic P-450 1A1 mRNA was consistent with other indices of induction such as $P-4501 \mathrm{~A} 1$ itself and EROD activity, but was less well correlated with tissue or ambient organic con- 
taminants. The hepatic enzyme activity CN-ECOD appeared as sensitive as EROD to ambient contaminants. MFO induction in kidney, but not in gill, was reasonably well correlated to environmental contamination.

(2) Hepatic MT induction did not bear any simple relationship to ambient metal or tissue metal concentrations. It appeared to respond differently in female (where it was best correlated with $\mathrm{Zn}$ ) and male dab (best correlated with $\mathrm{Cd}$ and $\mathrm{Cu}$ ), and to be affected by both exogenous and endogenous factors. MT induction in gill (from male dab) was more clearly related to metal exposure, especially $\mathrm{Zn}$.

(3) AChE and BChE inhibition followed roughly the same trend as that of MFO induction, and suggested that the chemicals responsible for cholinesterase inhibition were distributed similarly to chlorinated or polynuclear aromatic hydrocarbons. These cholin- esterase inhibitors should be identified and analysed in future studies.

(4) Measurement of ATPase inhibition and antioxidant enzyme activities showed some interesting responses to tissue or ambient contaminant concentrations. Although there are good theoretical grounds for considering these measurements as candidate approaches to 'effects monitoring', their basic biochemistry must be better defined.

(5) Finally, some obvious questions remain, the most important of which are:

(i) What are the processes and factors affecting metal accumulation and tissue distribution in dab, and how do these affect MT induction?

(ii) What cholinesterase inhibitors are present in the marine environment? Do these include the 'traditional' carbamate and organophosphorus pesticides, or are unexpected inhibitors present? 